\title{
Defining training needs, core competencies and future certification for Canadian hospitalists
}

\author{
Scott D. Smith MD MSc, Khalil Sivjee MD
}

$\mathrm{T}$ he Canadian hospitalist movement has rapidly grown from an emerging trend to a widespread reality. From coast to coast, hospitalists are here, most of them family physicians. ${ }^{1}$ A recent national survey showed that there are currently more than 100 hospitalist groups in Canada, with individual hospitalists caring for an average of 15 patients per eight-hour shift. ${ }^{2}$

Unfortunately, despite the rapid growth of this new specialty, remarkably little attention has been paid to the effect Canadian hospitalists have on quality outcomes, or to defining the training, certification, or the core skills they require to practise hospital medicine.

The need to define the training requirements of hospitalists and their scope of practice is not unique to the Canadian health care system. The United States has substantial experience in this regard. There, the hospitalist model is especially robust, with more than 20000 hospitalists, mostly internists, practising today. ${ }^{3}$ Hospital medicine is recognized in the US as the newest of the site-defined specialties, following in the footsteps of critical care medicine and emergency medicine. ${ }^{4}$

American hospitalists have been proactive in defining their scope of practice, developing core competencies and partnering with hospitals to improve quality of care and patient safety. Recently, the American Board of Internal Medicine created a new program that provides internists who practise in the hospital setting the option to maintain certification in internal medicine with a focused practice in hospital medicine. Parallel developments have not occurred in Canada. There are few training opportunities beyond the residency in family medicine, no Canadian core competencies have been developed, and no certification process has been established.

To better understand the differences in the evolution of hospital medicine between Canada and the US, one must appreciate the events that led to the adoption of the hospitalist model in Canada. The emergence of Canadian family physicians being employed as hospitalists was precipitated by several socioeconomic factors. The sentinel event was a shortage of family physicians in the 1990s, which led to a large number of "orphan patients" arriving at hospitals (i.e., patients without a family physician of their own to admit and care for them). Family physicians who previously admitted their own patients to hospital were expected to manage this high volume of orphan patients. Many were unwilling to accept this added workload and resigned their hospital privileges. These factors, coupled with inadequate remuneration for inpatient care and the increasing complexity and acuity of elderly patients admitted to hospital, caused an even greater number of family physicians to withdraw their hospital privileges. ${ }^{5}$ Concurrently, increasing workloads providing outpatient primary care meant family physicians no longer had the time to provide both inpatient and outpatient care. ${ }^{5}$ The result was a critical mass of unassigned patients in hospitals.

In response to this crisis, hospitals recruited and employed family physicians as hospitalists. This novel career path was attractive because it provided an opportunity for family physicians to practice higher-acuity medicine while earning competitive financial compensation and maintaining a good work-life balance. ${ }^{2}$ Because the Canadian hospitalist movement appeared independently, at the level of individual hospitals, the scope of clinical activities was not predefined and there was no national discussion as to the training or certification that would be necessary.

Residents in family medicine in Canada complete a two-year training program; in every other industrialized country, family physicians complete a minimum of three years of training. ${ }^{6}$ During this two-year period, residents spend only two to three months managing the care of patients on inpatient wards. Within the current

\section{KEY POINTS}

- There has been rapid growth in the number of Canadian hospitalist programs in the past decade.

- In contrast to the United States, Canadian hospitalists are primarily family physicians.

- Substantial work remains to be done to clearly define the training needs, core skills and potential certification of Canadian hospitalists. 
training structure, reviewers of a large academic training program in family medicine recently noted that residents may not encounter some common inpatient conditions, and opportunities to develop procedural skills are incomplete. ${ }^{?}$

Given these realities, should we have formalized training programs to prepare family physicians who are interested in pursuing careers as hospitalists? Precedents for additional training exist in anesthesia, aboriginal health, emergency medicine, elder care, obstetrics and palliative care.

The issues of training and certification have begun to be addressed, but the process is still in its infancy. A small number of voluntary training opportunities have emerged. An informal survey of leading hospitalists from across Canada identified two training programs in Ontario (both in Toronto), and one program currently in the planning stages in British Columbia. In addition, the College of Family Physicians of Canada recently approved the creation of a hospital medicine program within the section of family physicians with special interests or focused practices. The development of further training programs will pose many challenges to leaders in hospital medicine. One concern is that if family physicians choose careers dedicated to hospital medicine, fewer of them will be available to offer comprehensive primary care in the community.

A challenge to the career development of Canadian hospitalists is the lack of a formal assessment to evaluate requisite core skills and scope of practise. A potential template exists from the American Society of Hospital Medicine's Core Competencies in Hospital Medicine. The three major sections of this document include "Clinical Conditions" (the most common acute medical problems hospitalists may encounter, such as congestive heart failure, chronic obstructive pulmonary disease and stroke), "Procedures" (such as paracentesis and lumbar puncture) and "Healthcare Systems" (including interprofessional collaboration, safe patient handover and informed discharge planning). ${ }^{8}$

In addition to defining training requirements and core competencies, the impact of hospitalists on quality of care will also need to be evaluated using validated metrics such as the hospitalized standardized mortality ratio. Studies in the US have shown that hospitalists reduce patients' length of stay, while providing modest cost savings without negatively affecting quality measures. ${ }^{9}$ No comparable studies exist evaluating the impact of Canadian hospitalists.
The future of hospitalists in Canada remains unclear; remuneration and relationships with local hospital leadership are in flux. What is certain is that the "silver tsunami" of aging baby boomers will soon crash through the doors of Canadian hospitals. Providing high-quality and efficient care for these patients will require unprecedented levels of resources and place tremendous strain on our health care system. Greater dialogue between the College of Family Physicians of Canada, the Canadian Society of Hospital Medicine, provincial ministries of health, hospitals, hospitalist groups and patient advocacy groups is needed to decide how hospitalists will help meet this imminent challenge. Clearly, this discussion must address future training needs, certification, core competencies and scope of practice for hospitalists, as well as methods of measuring the impact and quality of the care they deliver.

\section{References}

1. Canadian hospitalist demographics. Vancouver (BC): Canadian Society of Hospital Medicine; 2008. Available: http://canadianhospitalist.ca /content/canadian-hospitalist-demographics. (accessed 2011 Nov. 3).

2. CSHM survey results. Vancouver (BC): Canadian Society of Hospital Medicine; 2008. Available: http://canadianhospitalist.ca /content/cshm-survey-results (accessed 2011 Nov. 3).

3. 2005-2006 CSHM survey: the authoritative source on the state of hospital medicine: highlights/executive summary. Vancouver (BC): Canadian Society of Hospital Medicine; 2006. Available: www.hospitalmedicine.org/AM/Template.cfm?Section=Survey $\&$ Template $=/ \mathrm{CM} /$ ContentDisplay.$c f m \&$ ContentID=14352 (accessed 2011 Nov. 3).

4. Wachter RM, Goldman L. The hospitalist movement 5 years later. JAMA 2002;287:487-94.

5. Family physicians caring for hospital inpatients: a discussion paper prepared by The College of Family Physicians of Canada. Mississauga ( $\mathrm{ON})$ : The College of Family Physicians of Canada; 2003. Available: www.cfpc.ca/uploadedFiles/Resources /Resource_Items/FPs20Inpt20Hosp20Care_En.pdf (accessed 2011 Jan. 24).

6. Lehmann F. Should family medicine residency be 3 years? YES. Can Fam Physician 2009;55:342-4.

7. Jugovic P, Sivjee K, Mistry R. Hospitalist training programs. Refining the Hospitalist Model: Stability and Integration; 2008 Feb. 28-29; Toronto (ON): Insight Information; 2008.

8. The core competencies in hospital medicine: a framework for curriculum development by the society of hospital medicine. $J$ Hosp Med 2006;1(Suppl 1):2-95.

9. Lindenauer PK, Rothberg MB, Pekow PS, et al. Outcomes of care by hospitalists, general internists, and family physicians. $N$ Engl J Med 2007;357:2589-600.

Affiliations: Scott Smith is with the Kelowna General Hospital, Kelowna, BC; Khalil Sivjee is with the Hospitalist Training Program, Sunnybrook Health Sciences Centre, Toronto, Ont.

Contributors: Both authors worked jointly in discussions around the conception and goals of the commentary, in writing and editing the commentary and in approving the final version of the accepted manuscript.

Acknowledgements: The authors thank Kaveh Shojania and Wendy Levinson for their helpful comments. 